\title{
Special issue on soft computing and intelligent systems: Tools, techniques and applications
}

\author{
Sabu M. Thampi ${ }^{\mathrm{a}, *}$ and El-Sayed M. El-Alfy ${ }^{\mathrm{b}}$ \\ ${ }^{\mathrm{a}}$ Indian Institute of Information Technology and Management - Kerala (IIITM-K), Technopark Campus, \\ Trivandrum, Kerala, India \\ ${ }^{\mathrm{b}}$ Department of Information and Computer Science, College of Computer Sciences and Engineering, \\ King Fahd University of Petroleum and Minerals, Dhahran, Saudi Arabia
}

This special issue is a selected collection of papers submitted to the Second International Symposium on Intelligent Systems Technologies and Applications (ISTA'16), September 21-24, 2016, Jaipur, India. These papers have been reviewed and accepted for presentation at the symposium and for publication in the Journal of Intelligent \& Fuzzy Systems (JIFS). In this special issue there are 44 papers covering a wide range of tools, techniques and applications of soft computing and intelligent systems.

The International Symposium on Intelligent Systems Technologies and Applications aims to bring together researchers in related fields to explore and discuss various aspects of intelligent systems technologies and their applications. It provides excellent opportunities for the presentation of interesting new research results and discussion about them, leading to knowledge transfer and generation of new ideas.

There are seven papers addressing research problems in bioinfomratics and medical research. In [1], a classification system that combines feature selection techniques with semi-supervised fuzzy

${ }^{*}$ Corresponding author. Sabu M. Thampi, Indian Institute of Information Technology and Management - Kerala (IIITMK), Technopark Campus, Trivandrum 695581, Kerala, India. Tel.: +91 471 2784117; Fax: +91 471 2527568; E-mail: sabu.thampi@iiitmk.ac.in. c-means (FCM) algorithm is proposed and evaluated on publicly available gene expression datasets. The results show that even with a few labeled samples, accurate prediction of cancer subtypes can be attained. In [2], a new texture-based feature extraction algorithm is proposed for extracting relevant and informative features from brain MR images having tumor. It is based on finding the texture description using nine different variants of texture objects and forming an intermediate texture index matrix using texture objects with high-pass and low-pass spiral filters. The resultant two-index matrix is used to generate the Texture Co-occurrence Matrix which helps to extract spatial and spectral domain features for brain MRI classification. Experiments on a dataset of $660 \mathrm{~T} 1$-weighted post contrast brain MR images with five different types of malignant tumors show that the proposed method can lead to significant results in abnormality classification when compared with the state-of-the-art GLCM and Run-length algorithms. In [3], a novel method is proposed for early detection and diagnosis of breast cancer in digital mammograms. The proposed technique uses Particle Swarm Optimization (PSO), Cellular Neural Network (CNN) and Probabilistic Neural Network (PNN). The breast mass texture feature extraction is carried out using Gray-Level Co-occurrence Matrix (GLCM) 
and the optimal texture features are selected using a particle swarm optimised feature selection method. A simple, robust retinal vessel extraction approach is described in [4] based on line detectors and morphological operations. The proposed algorithm overcomes the fundamental issues of scale and orientation with improved values of performance measures as compared to the-state-of-the-art techniques. In [5], a hybrid classifier with region-dependent integrated features is presented for automatic detection of one of the major eye diseases called diabetic retinopathy (DR). This approach combines holoentropy-enabled decision tree with a feed-forward neural network using score-level fusion. The performance is evaluated on two different databases. In [6], a computer-aided diagnosis system is presented to quantify erythrocytes in microscopic images of thin blood smears. The proposed method consists of preprocessing, segmentation, morphological filtering, cell separation and clump cell segmentation. Geometric features such as cell area, compactness ratio and aspect ratio have been used to define the feature set and different classifiers have been used and evaluated. In [7], a hardware efficient reconfigurable denoising system is proposed for real-time electrooculography (EOG) biomedical signal processing.

Cyber security is another vital area of research and in this special issue there are six papers dealing with related issues including privacy, authentication, and intrusion detection. In [8], a genetic Bottom-Up Generalization (BUG) and Top-Down Specialization (TDS) model with pseudo-identity is presented to accomplish privacy preservation over incremental data sets in cloud storage. The data sets (DS) are partitioned in the preprocessing stage then clustered as groups. The genetic model is used for indexing and updating the incremental data sets. This hybrid approach shows the synergy of various privacy preservation techniques which offer enough tradeoff among data utility and privacy protection. In [9], the authors presented a novel multi-class approach for network intrusion detection based on a cascade ensemble of artificial neural network. The cascade structure uses the trained neural network to filter the training data which is used along with a boostingbased learning algorithm to learn an optimal set of neural network parameters for each successive partition. In [10], a new cryptographic method is proposed for image encryption. This approach is based on shuffling pixels and changing their values using 128-bit secret key and Henon chaotic map. Pixel shuffling is performed via horizontal and vertical permutation to expand diffusion in the image and dissipate the high correlation among image pixels. Results show that the proposed approach is highly key sensitive and has a good resistance against various brute-force and statistical attacks. In [11], a novel adaptive histogram equalization (AHE) variant is proposed and applied to biometric authentication. It is evaluated on IITD palmprint databases of left and right hands. Identifying traffic anomalies is a challenge, as it requires mining and identifying patterns among a huge volume of data. In [12], statistical analysis, supervised learning and ensemble based dynamic reputation are used to identify benign and abnormal domains receiving huge volumes of traffic within a short period of time. In [13], another system is presented to detect attacks in both network and virtualization layers in cloud computing. The authors called it "Program Semantic-Aware Intrusion Detection at Network and Hypervisor Layer (PSINetVisor)". It applies dynamic analysis and machine learning approaches to learn the behavior of anomalies to be secure from obfuscation based attacks. The proposed system is validated on the latest intrusion dataset (UNSW-NB) and malware binaries.

Another crucial area in computer science with immense number of publications is natural language processing. Four papers in this special issue deal with prominent feature extraction in question-answering systems, automated processing and recognition of human speech commands, document analysis and categorization, and microblogging analysis and hierarchical visualization. In [14], a prominent feature extraction for evidence gathering and scoring in question answering is presented. Various features including lexical, syntactic, semantic and structural features have been investigated for learning answer evidence. In [15], a low-level word processing system for recognizing isolated words is proposed using mean-variance normalized frequency-time spectrograms and a minimum distance similarity metric. This method is evaluated and compared with DTW on TIMIT database with promising results in terms of improved recognition accuracies, robustness to noise, lower computational requirements, and scalability to large world problems. In [16], a semantics-based probabilistic model is introduced for generating a reduced representation of a large collection of documents. Documents are processed by tokenization, pronoun resolution, word collocation, synonym linking, named entity recognition, tagging, lemmatizing and word sense disambiguation. More meaningful topics are generated by Latent Dirichlet Allocation 
(LDA). Microblogging services like Twitter have gained immense popularity. In [17], a framework for event detection is described using recursive hierarchical clustering. The different levels of the hierarchy represent the events at different time granularities.

Enriching learning environments with intelligent interactive applications attracted increasing attention of researchers to facilitate learning at the student pace and address special situations. There are three related papers to this field in this special issue. In [18], an elearning system architecture is described. It receives gesture triggers and dynamically adapts to interaction patterns of participants facilitating eye contact. In [19], some interesting ideas for an interactive social companion for children with Autism Spectrum Disorder (ASD), called Aarya, are presented. The tool provides gesture-based virtual environment and helps children to improve their confidence in forming relationships and interacting with other people. In [20], the author presents a case study for investigating sequential adaptive testing using real-data simulation. Administering such sequential adaptive tests has many benefits including personalized tests, accurate measurement, item security, and substantial cost reduction. However, the design of such intelligent tests is a complex process and it is important to explore the impact of various parameters and options on the performance before switching from traditional tests in a particular environment. Throughout several experiments, the performance of a number of sequential adaptive testing procedures is evaluated and compared using various criteria.

Another important area in data mining is recommendation systems which has gained popularity with the proliferation of online data. In [21], a communitybased collaborative filtering recommender system is described. It utilizes Louvain method to discover communities in the dataset. Recommendations are generated based on the idea of Item FrequencyInverse Community Frequency (IF-ICF) scores. The authors have developed and evaluated a prototype of the system using Java. In [22] the authors propose a content-based recommender system using Hadoop MapReduce, which is a scalable, fault tolerant and distributed framework. This system is based on user profiling and document feature extraction using a vector-space model followed by computing similarity to generate recommendations for the target user. In [23], a web page recommendation system is proposed based on partially ordered sequential association rules in making future predictions to facilitate web browsing. Two sequential rule-mining algorithms have been investigated.

Five papers in this special issue address a number of applications of soft computing and fuzzy control in industrial and power systems. One problem faced by power systems is related to voltage instability and chaos. A Perturb-Boost Fuzzy-Logic Controller is described in [24] for instability control in nonlinear dynamical systems. It is based on injecting reactive power through rule-based design based on voltage collapse magnitude to mitigate the chaos and instability trajectories. There are many applications for the presented technique in industrial control, mechanical and electrical nonlinear systems and systems governed by nonlinear differential equations. A simulink model of the proposed technique is described and evaluated for a fourth-order model of a power system subjected to mechanical power ripples in turbine output causing instability. In [25], a learning automata algorithm is applied for the optimization of a Fuzzy Maximum Power Point Tracking (MPPT) controller for a solar photovoltaic (PV) system under fast changing environmental conditions. A prototype for the proposed system is developed and tested in Matlab for various environmental conditions. In [26], an intelligent model is proposed for fault diagnosis of a transmission line. This method is based on Empirical Mode Decomposition (EMD) and artificial neural network (ANN). Features are extracted from nine different types of faults simulated along with healthy condition in a MATLAB-based transmission line system. Then, the most relevant features are selected and used for fault classification. In [27], a hybrid wavelet-ANN protective scheme is proposed for a multi-terminal Extra-High Voltage (EHV) transmission line having multiple series compensation. Statistical features are extracted using a Multi-Resolution Analysis (MRA) wavelet transform and relevant features are selected based on ANOVA F-test statistics. In [28], a fuzzy-based temperature monitoring system is described for a rotary kiln. The proposed approach integrates RGB intensities of a flame image as fuzzified inputs, temperature as defuzzified output variables and fuzzy inference Mamdani models.

With the massive evolution of today's information technology, scalability and effectively allocating and sharing resources including computing platforms and wireless spectrum are very active research areas in cloud computing, parallel processing, cognitive radio networks, wireless sensor network and Internetof-Things (IoT). In [29], the authors propose a 
"Cloud + Container" portal platform for Internet of Everything (IoE). The platform can provide the application development, deployment, operations functions for developers, and it is convenient for users to manage the mass applications. To tackle the energy and processing limitations of mobile phones, the authors in [30] propose a dynamic decisionmaking system based on statistical regression to allow smartphones to effectively offload computationally intensive tasks to the cloud. The integration of Internet of Things (IoT) and Web 2.0 for inventory management has been addressed in [31] taking advantages of both technologies. The proposed prototype is able to detect misplaced products, detect low stock levels and send notifications on Twitter to update the inventory manager on mobile phone. In [32], the authors review existing approaches for dynamic spectrum reconfiguration of cognitive radio networks and propose a biologically-inspired optimal foraging approach to address the spectrum decision making in a distributed cognitive radio network. In [33], a hybrid approach is proposed for cluster-head election in wireless sensor networks. It integrates energy and trust based cluster-head selection schemes for effective election of cluster head. In [34], an area efficient and high speed 32-bit floating point FFT processor for OFDM using Vedic multiplication process is presented. In [35], new parallel processor architectures have been used and a friendship-based load balancing strategy has been designed and implemented to optimally utilize the parallel processor components.

Five papers in this special issue address problems in signal, image and video processing systems. In [36], a wavelet fusion based on fuzzy-inference system is proposed to enlighten regions of a nonuniform illuminated night video surveillance system. This approach includes spatial and temporal illumination and generates a contribution index using a fuzzy membership function. Stationary wavelets are used to decompose high and low-frequency coefficients and contribution index selects the illuminated regions for fusion. Finally, the inverse wavelet transform is applied to reconstruct the illumination enhanced frame. In [37], a pan-sharpening technique is proposed based on M-band wavelet to improve the quality of a low-resolution multispectral image by fusion with a high-resolution panchromatic image. In [38], the authors propose an efficient and costeffective method to determine the traffic state of the road using acoustic data collected from the commuter's smartphone. They compare the accuracy of Support Vector Machines and Neural Networks by using data collected from different recording sessions. In [39], the authors propose a self-tuning adaptive filter algorithm for acoustic noise cancellation by addressing two important factors: step size and filter length. In [40], the authors propose a technique for detecting forged images based a compact multi-texture representation capable of capturing texture variations. Experimental results show that this technique performs better than any of the texture descriptors applied individually.

Evolutionary techniques are addressed in two of the papers in this special issue. In [41], the authors describe a novel harmony search algorithm to improve the performance of opposition-based learning (OBL) theory. The proposed approach considers each of the components of the candidate solution individually and attempts to find the best possible combination among them. In [42], the firefly algorithm is applied to an uncapacitated facility location problem.

Finally, in [43] the authors address a problem in data mining to find frequent itemsets using improvised Apriori algorithm with frequent subgraph tree. In [44], an intelligent earphone with auto-pause facility is described.

In conclusion, this special issue would not have been possible without the help of many people. As guest editors, we would like to take this opportunity to thank the authors for their contributions and the reviewers for their invaluable comments and timely responses. We also would like to thank the JIFS Editor-in-Chief and staff for their support during the preparation and production of this special issue.

\section{References}

[1] P.S. Deepthi and S.M. Thampi, Predicting cancer subtypes from microarray data using semi-supervised fuzzy c-means algorithm, Journal of Intelligent \& Fuzzy Systems (JIFS) 32 (2017), 2797-2805.

[2] A. Vidyarthi and N. Mittal, Texture based feature extraction method for classification of brain tumor MRI, Journal of Intelligent \& Fuzzy Systems (JIFS) 32 (2017), 2807-2818.

[3] P. Stephen, R. Subban, A. Devi and V. Jothimani, Particle swarm optimised computer aided diagnosis system for classification of breast masses, Journal of Intelligent \& Fuzzy Systems (JIFS) 32 (2017), 2819-2828.

[4] S.B. Patil, A.S. Narote and S.P. Narote, Efficient retinal vessel detection using line detectors with morphological operations, Journal of Intelligent \& Fuzzy Systems (JIFS) 32 (2017), 2829-2836.

[5] V.M. Mane, D.V. Jadhav and S.D. Shirbahadurkar, Hybrid classifier and region-dependent integrated features for 
detection of diabetic retinopathy, Journal of Intelligent \& Fuzzy Systems (JIFS) 32 (2017), 2837-2845.

[6] S.S. Devi, J. Singha, M. Sharma and R.H. Laskar, Erythrocyte segmentation for quantification in microscopic images of thin blood smears, Journal of Intelligent \& Fuzzy Systems (JIFS) 32 (2017), 2847-2856.

[7] S. Agarwal, V. Singh, A. Rani and A.P. Mittal, Hardware efficient denoising system for real EOG signal processing, Journal of Intelligent \& Fuzzy Systems (JIFS) 32 (2017), 2857-2862.

[8] K.C. Sreedhar, M.N. Faruk and B. Venkateswarlu, A genetic TDS and BUG with pseudo-identifier for privacy preservation over incremental data sets, Journal of Intelligent \& Fuzzy Systems (JIFS) 32 (2017), 2863-2873.

[9] M.M. Baig, M.M. Awais and E.-S.M. El-Alfy, A multiclass cascade of artificial neural network for network intrusion detection, Journal of Intelligent \& Fuzzy Systems (JIFS) 32 (2017), 2875-2883.

[10] K. Mishra, R. Saharan and B. Rathor, A new cryptographic method for image encryption, Journal of Intelligent \& Fuzzy Systems (JIFS) 32 (2017), 2885-2892.

[11] Gopal, S. Srivastava and S. Srivastava, Biometric authentication using local subspace adaptive histogram equalization, Journal of Intelligent \& Fuzzy Systems (JIFS) 32 (2017), 2893-2899.

[12] A. Ashok, P. Poornachandran, S. Pal, P. Sankar and K. Surendran, Why so abnormal? Detecting domains receiving anomalous surge traffic in a monitored network, Journal of Intelligent \& Fuzzy Systems (JIFS) 32 (2017), 2901-2907.

[13] P. Mishra, E.S. Pilli, V. Varadharajan and U. Tupakula, PSINetVisor: Program semantic aware intrusion detection at network and hypervisor layer in cloud, Journal of Intelligent \& Fuzzy Systems (JIFS) 32 (2017), 2909-2921.

[14] L.K. Sharma and N. Mittal, Prominent feature extraction for evidence gathering in question answering, Journal of Intelligent \& Fuzzy Systems (JIFS) 32 (2017), 2923-2932.

[15] M. Milacic, A.P. James and S. Dimitrijev, Recognizing isolated words with minimum distance similarity metric padding, Journal of Intelligent \& Fuzzy Systems (JIFS) 32 (2017), 2933-2939.

[16] R.R.K. Menon, D. Joseph and M.R. Kaimal, Semanticsbased topic inter-relationship extraction, Journal of Intelligent \& Fuzzy Systems (JIFS) 32 (2017), 2941-2951.

[17] N. Akhtar and B. Siddique, Hierarchical visualization of sport events using Twitter, Journal of Intelligent \& Fuzzy Systems (JIFS) 32 (2017), 2953-2961.

[18] N. Ramkumar, P.V. Rangan, U. Gopalakrishnan and B. Hariharan, Gesture triggered, dynamic gaze alignment architecture for intelligent learning systems, Journal of Intelligent \& Fuzzy Systems (JIFS) 32 (2017), 2963-2969.

[19] R. Sreedasyam, A. Rao, N. Sachidanandan, N. Sampath and S.K. Vasudevan, Aarya - A Kinesthetic companion for children with Autism Spectrum Disorder, Journal of Intelligent \& Fuzzy Systems (JIFS) 32 (2017), 2971-2976.

[20] E.-S.M. El-Alfy, Evaluation of sequential adaptive testing with real-data simulation: A case study, Journal of Intelligent \& Fuzzy Systems (JIFS) 32 (2017), 2977-2986.

[21] C. Sharma and P. Bedi, CCFRS - Community based Collaborative Filtering Recommender System, Journal of Intelligent \& Fuzzy Systems (JIFS) 32 (2017), 2987-2995.

[22] A. Gautam and P. Bedi, Developing content-based recommender system using Hadoop Map Reduce, Journal of Intelligent \& Fuzzy Systems (JIFS) 32 (2017), 2997-3008.

[23] H. Singh, M. Kaur and P. Kaur, Web page recommendation system based on partially ordered sequential rules,
Journal of Intelligent \& Fuzzy Systems (JIFS) 32 (2017), 3009-3015.

[24] S.P. Nangrani and S.S. Bhat, Instability, chaos and bifurcation control in nonlinear dynamical system behavior using perturb-boost fuzzy logic controller, Journal of Intelligent \& Fuzzy Systems (JIFS) 32 (2017), 3017-3029.

[25] S.S. Mohammed, D. Devaraj and T.P.I. Ahamed, Learning automata based fuzzy MPPT controller for solar photovoltaic system under fast changing environmental conditions, Journal of Intelligent \& Fuzzy Systems (JIFS) 32 (2017), 3031-3041.

[26] H. Malik and R. Sharma, EMD and ANN based intelligent fault diagnosis model for transmission line, Journal of Intelligent \& Fuzzy Systems (JIFS) 32 (2017), 3043-3050.

[27] P.D. Raval and A.S. Pandya, A hybrid Wavelet-ANN protection scheme for series compensated EHV transmission line, Journal of Intelligent \& Fuzzy Systems (JIFS) 32 (2017), 3051-3058.

[28] L.R. Sreedhanya, A. Varghese, M.S. Nair and M. Wilscy, Temperature mapping of a rotary kiln using fuzzy logic, Journal of Intelligent \& Fuzzy Systems (JIFS) 32 (2017), 3059-3067.

[29] X. Chen, P. Yang, T. Qiu, H. Yin and J. Ji, IoE-MPP: A mobile portal platform for internet of everything, Journal of Intelligent \& Fuzzy Systems (JIFS) 32 (2017), 3069-3080.

[30] N.M. Dhanya, G. Kousalya and P. Balakrishnan, Dynamic mobile cloud offloading prediction based on statistical regression, Journal of Intelligent \& Fuzzy Systems (JIFS) 32 (2017), 3081-3089.

[31] S. Mathaba, M. Adigun, J. Oladosu and O. Oki, On the use of the Internet of Things and Web 2.0 in inventory management, Journal of Intelligent \& Fuzzy Systems (JIFS) 32 (2017), 3091-3101.

[32] O.A. Oki, T.O. Olwal, P. Mudali and M. Adigun, Dynamic spectrum reconfiguration for distributed cognitive radio networks, Journal of Intelligent \& Fuzzy Systems (JIFS) 32 (2017), 3103-3110.

[33] P.L. Rajarajeswari and N.K. Karthikeyan, Hyper-geometric energy factor based semi-Markov prediction mechanism for effective cluster head election in WSNs, Journal of Intelligent \& Fuzzy Systems (JIFS) 32 (2017), 3111-3120.

[34] B. Kalra and J.B. Sharma, Vedic multiplication based efficient OFDM FFT processor, Journal of Intelligent \& Fuzzy Systems (JIFS) 32 (2017), 3121-3128.

[35] R.K. Bhullar, L. Pawar, R. Bajaj and A.K. Manocha, Intelligent stress calculation and scheduling in segmented processor systems using buddy approach, Journal of Intelligent \& Fuzzy Systems (JIFS) 32 (2017), 3129-3142.

[36] T. Soumya and S.M. Thampi, A fuzzy fusion approach to enlighten the illuminated regions of night surveillance videos, Journal of Intelligent \& Fuzzy Systems (JIFS) 32 (2017), 3143-3149.

[37] V.V. Pradeep, V. Sowmya and K.P. Soman, Application of M-band wavelet in pan-sharpening, Journal of Intelligent \& Fuzzy Systems (JIFS) 32 (2017), 3151-3158.

[38] A. Kaur, N. Sood, N. Aggarwal, D. Vij and B. Sachdeva, Traffic state detection using smartphone based acoustic sensing, Journal of Intelligent \& Fuzzy Systems (JIFS) 32 (2017), 3159-3166.

[39] M. Dewasthale and R.D. Kharadkar, High performance self tuning adaptive filter algorithm for noise cancellation in speech, Journal of Intelligent \& Fuzzy Systems (JIFS) 32 (2017), 3167-3176.

[40] D.S. Vidyadharan and S.M. Thampi, Digital image forgery detection using compact multi-texture representation, 
Journal of Intelligent \& Fuzzy Systems (JIFS) 32 (2017), 3177-3188.

[41] R. Sarkhel, T.M. Chowdhury, M. Das, N. Das and M. Nasipuri, A novel Harmony Search algorithm embedded with metaheuristic Opposition Based Learning, Journal of Intelligent \& Fuzzy Systems (JIFS) 32 (2017), 3189-3199.

[42] K. Tsuya, M. Takaya and A. Yamamura, Application of firefly algorithm to the uncapacitated facility location problem, Journal of Intelligent \& Fuzzy Systems (JIFS) 32 (2017), 3201-3208.
[43] J.J. Nair and S. Thomas, Improvised Apriori with frequent subgraph tree for extracting frequent subgraphs, Journal of Intelligent \& Fuzzy Systems (JIFS) 32 (2017), 3209-3219.

[44] S.N. Abhishek, R.B. Balakirithikaa, C. Madhan and S.K. Vasudevan, An innovative and intelligent earphone with auto pause facility, Journal of Intelligent \& Fuzzy Systems (JIFS) 32 (2017), 3221-3228. 